\title{
Pesquisa sobre Criatividade em Contabilidade Gerencial: Visão Geral e Oportunidades de Pesquisa no Contexto Brasileiro
}

\author{
Andson Braga de Aguiar \\ https://orcid.org/0000-0003-4034-4134 | E-mail:abraga@usp.br \\ Ricardo Suave \\ https://orcid.org/0000-0002-9149-2658 | E-mail: ricardosuave@outlook.com
}

Criatividade representa fonte de vantagem competitiva para organizações e, por esse motivo, tem sido tema amplamente pesquisado em psicologia e administração (Speckbacher, 2017). Apesar da importância do tema, a ênfase predominante da pesquisa em contabilidade gerencial tem sido em examinar efeitos de sistemas de controle gerencial sobre desempenho dos indivíduos em tarefas rotineiras, ou seja, tarefas programáveis e repetítiveis (Brüggen, Feichter $\&$ Williamson, 2018). Apenas recentemente, a pesquisa em contabilidade gerencial começa a se preocupar com o efeito de controles gerenciais no desempenho em tarefas criativas as quais são de mais difícil previsibilidade e são não repetítiveis (Shields, 2015). No contexto brasileiro, em particular, estudos em contabilidade gerencial com ênfase em criatividade ainda são inexistentes. A importância em se entender o papel de controles gerenciais para estimular criatividade no ambiente de trabalho é permitir que sistemas de controle gerencial sejam desenhados de modo apropriado ao nível de criatividade desejado pelas organizações.

O propósito deste editorial é oferecer uma visão geral da pesquisa sobre criatividade em contabilidade gerencial e discutir oportunidades para pesquisas futuras no contexto brasileiro. Em particular, pretendese abordar às seguintes questões: Como criatividade tem sido definida e operacionalizada na pesquisa em contabilidade gerencial? Controle e criatividade representam objetivos conflitantes? Quais controles gerenciais são examinados para explicar criatividade? Quais oportunidades de pesquisa existem sobre o tema no Brasil? A discussão em torno dessas questões é feita a partir de pesquisadores que têm dedicado particular atenção ao tema de criatividade, tais como, Michael Williamson, Isabela Grabner e Gerhard Speckbacher, em contabilidade gerencial, e Teresa Amabile e Robert Eisenberger, em psicologia e gestão.

\section{Conceitos e Operacionalização de Criatividade}

Existe falta de consenso sobre a definição de criatividade nas pesquisas sobre o tema (Amabile, 1983). Devido a tal problema, Amabile (1996) sugere a adoção de duas definições complementares, uma conceitual e outra consensual. A definição conceitual foca no processo e, portanto, entende criatividade como um processo que resulta em algo inovador e útil a um grupo em algum momento do tempo. Já a abordagem consensual, predominante na pesquisa sobre o tema, foca no produto e, desse modo, especifica que um produto é criativo quando especialistas, de maneira independente, definem-no como criativo. Enquanto a visão de processo é necessária para construção de uma teoria da criatividade, a visão de produto é importante para desenvolvimento de métodos de avaliação (Amabile, 1996). 
Percebem-se, portanto, dois aspectos principais dessas duas definições de criatividade: o primeiro caracterizado por termos como inovador e diferente, enquanto o segundo por termos como aceitabilidade, adequação e utilidade. Assim, para que algo seja considerado criativo, deve ser inovador em relação ao que se tem até o momento, mas tal novidade precisa ainda ser útil, aplicável. Vale destacar que, embora exista relação entre criatividade e inovação, esses dois conceitos são diferentes, pois criatividade, juntamente com implementação, são componentes necessários à inovação (Axtell et al., 2000; Shalley \& Gilson, 2004).

Assim como ocorre com a pesquisa sobre criatividade em psicologia e administração, predomina na pesquisa em contabilidade gerencial a definição de criatividade como produto. Embora enfatizando a definição como produto, a pesquisa sobre criatividade em contabilidade gerencial tem seguido até o momento duas abordagens distintas principais para tratar o tema, seja em termos de abordagem teórica, seja em termos de desenho de pesquisa.

De um lado, os estudos de Williamson e seus coautores (p. ex., Kachelmeier, Reichert e Williamson, 2008; Kachelmeier \& Williamson, 2010; Brüggen et al., 2018) empregam uma abordagem de agência e utilizam desenhos experimentais para examinar o efeito de diferentes contratos de incentivo sobre desempenho em tarefas criativas. Nesses estudos, criatividade é definida como produção de ideias originais que podem contribuir para inovação, adaptação e crescimento organizacional (Kachelmeier et al., 2008). Em termos operacionais, Williamson e seus coautores têm utilizado diferentes variações para capturar criatividade dos participantes, com destaque para desenho de rebus puzzles (Kachelmeier et al., 2008; Kachelmeier \& Williamson, 2010; Kachelmeier, Wang \& Williamson, 2019), uso criativo para uma casa abandonada no campus da universidade (Chen, Williamson \& Zhou, 2012) e desenvolvimento e breve descrição de novas tarefas experimentais (Brüggen et al., 2018). Em geral, o desempenho de participantes em tarefas criativas é avaliado por 'especialistas' que são informados terem sido os participantes solicitados a criar algo novo, inovador e útil. Esse procedimento está diretamente vinculado à definição de criatividade como produto (abordagem consensual), em que a indicação do que é criativo é feita por especialistas (Amabile, 1996).

De outro lado, os estudos de Grabner (2014; Grabner \& Speckbacher, 2016) partem de teorias organizacionais e empregam abordagem contingente para examinar, por meio de dados de levantamento, os controles gerenciais que organizações européias empregam para estimular criatividade. Em particular, Grabner define criatividade como a importância que organizações atribuem à criatividade de seus colaboradores como fonte de vantagem competitiva, sendo organizações que atribuem maior importância denominadas de 'dependentes de criatividade'. Em termos operacionais, Grabner desenvolve uma escala para mensurar o nível de dependência de criatividade das organizações a partir de cinco perguntas. Tal escala possui como base entrevistas com especialistas da área e apresenta assertivas relacionadas à criatividade como fonte de criação de valor e receitas, além do sucesso comercial e vantagem competitiva como dependentes da criatividade dos colaboradores.

Enquanto nos estudos iniciais essas duas principais abordagens de pesquisa sobre criatividade em contabilidade gerencial parecem caminhar de modo separado, estudos mais recentes começam a reconciliar as evidências obtidas por cada abordagem (p. ex., Kachelmeier et al., 2019) e a ampliar o escopo de pesquisa sobre o tema. Nessa direção, Speklé, van Elten e Widener (2017) se fundamentam em teorias organizacionais e definem criatividade como geração de novas ideias que facilitam a adaptação necessária para que organizações possam sobreviver e competir em mercados em constantes mudanças. Operacionalmente, Speklé et al. (2017) capturam criatividade por meio de levantamento junto a unidades de negócios de organizações européias em que respondentes autoavaliam o quanto eles podem desenvolver novas ideias e encontrar soluções para problemas associados ao desempenho de suas tarefas. 
É possível afirmar ainda que, embora exista um foco na definição de criatividade como produto, estudos mais recentes em contabilidade gerencial começam a considerar criatividade como processo. Por exemplo, Kachelmeier et al. (2019) definem criatividade em seu estudo experimental como um processo que inclui os seguintes estágios: preparação inicial, incubação e ganhos eventuais decorrentes da criatividade. Adicionalmente, destaquem-se os estudos de Cools, Stouthuysen e Van den Abbeele (2017) e de Davila e Ditillo (2017) que também adotam uma visão de processo, mas que empregam uma abordagem qualitativa para examinar o papel de mecanismos de controle gerencial no estímulo à criatividade. Cools et al. (2017) introduzem uma perspectiva multidimensional de criatividade em que são diferenciadas duas dimensões a depender do tipo de problema. De um lado, tem-se a criatividade esperada para lidar com problemas 'abertos' e 'autodescobertos'. De outro lado, tem-se a criatividade responsiva para lidar com problemas 'fechados' e 'que se apresentam. Por sua vez, Davila e Ditillo (2017) também entendem criatividade sob uma perspectiva multidimensional que inclui criatividade divergente e criatividade convergente. Enquanto criatividade divergente está associada à geração de ideias sem qualquer tipo de julgamento, criatividade convergente está associada à avaliação das ideias geradas na fase anterior (Basadur \& Finkbeiner, 1985). Em particular, o estudo de Davila e Ditillo (2017) examina criatividade convergente a qual incorpora uma inovação que enfatiza menos conhecimento técnico para desenvolvimento do produto.

Em síntese, percebe-se que a pesquisa sobre criatividade em contabilidade gerencial tem ampliado o seu escopo em termos de uma crescente variedade de definições para criatividade, com consequente uso de diferentes formas de operacionalizar essas definições. De outra forma, significa dizer que ainda não há uma abordagem predominante e mais aceita de definir e operacionlizar criatividade na literatura em contabilidade gerencial sobre o tema, podendo essa definição e operacionalização ser específica a cada contexto de pesquisa (Speckbacher, 2017).

\section{Existe Tensão entre Controle x Criatividade?}

Uma questão frequente na pesquisa sobre criatividade em contabilidade gerencial é se uso mais intenso de controles e estímulo à criatividade são objetivos conflitantes (Speckbacher, 2017). Em particular, a questão é se organizações que mais dependem de criatividade de seus colaboradores são também aquelas que deveriam fazer menos uso de controles gerenciais para monitorá-los e estimulá-los (Grabner \& Speckbacher, 2016). Estudos sobre criatividade costumam favorecer a perspectiva de incompatibilidade entre controle e criatividade (Amabile, 1982; 1983). Os dois principais argumentos em favor dessa perspectiva têm por base, de um lado, a hipótese de motivação intrínseca da criatividade e, de outro lado, o fato de que tarefas criativas possuem especificidades que tornam os mecanismos de controle de pouca utilidade ou mesmo contraproducentes (Speckbacher, 2017; Grabner \& Speckbacher, 2016).

Sob o ponto de vista do primeiro argumento, menciona-se que criatividade é conduzida por um estado de motivação intrínseca, enquanto motivação extrínseca inibe criatividade (Amabile \& Pillemer, 2012). Como afirma Speckbacher (2017), uma vez que muitos dos mecanismos de controle, tais como definição de metas, controles orçamentários e remuneração baseada em desempenho, são usados com o propósito de afetar a motivação extrínseca dos indivíduos, a expectativa é de que uso de controles gerenciais resulte em menor criatividade. 
Entretanto, a hipótese de motivação intrínseca tem sido desafiada por pesquisadores que sugerem que fatores extrínsecos (p. ex., incentivos) podem facilitar e estimular criatividade (p. ex., Eisenberger \& Cameron, 1996; Eisenberger \& Rhoades, 2001). Por exemplo, Eisenberger e Shanock (2003) sugerem ser possível alcançar motivação para tarefas criativas vinculando remuneração a desempenho em criatividade. Na pesquisa em contabilidade gerencial, diversos estudos (p. ex., Brüggen et al., 2018; Webb, Williamson \& Zhang, 2013; Kachelmeier \& Williamson, 2010) demonstram que contratos de incentivo podem facilitar o desempenho em tarefas criativas. Por exemplo, Kachelmeier et al. (2008) indicam ser possível estimular criatividade na presença de contratos de incentivo relativamente ao uso de salário fixo. Em outro exemplo, Grabner (2014) demonstra que para organizações dependentes de criatividade, remuneração baseada em desempenho é um importante controle para estimular criatividade de forma produtiva quando complementada por uma avaliação subjetiva de desempenho.

Quanto ao segundo argumento, tarefas criativas são vistas como contendo elevado nível de incerteza, seja em termos de quantidade de recursos necessários, ações esperadas, eficiência no uso de recursos ou em termos da probabilidade de obtenção de algum resultado a partir de tarefas criativas (p. ex., Amabile, 1982; 1983). Diante desses desafios de mensuração, controles gerenciais seriam inapropriados para estimular criatividade (Grabner \& Speckbacher, 2016).

Novamente, evidências empíricas nem sempre oferecem suporte para esse segundo argumento (Speckbacher, 2017; Adler \& Chen, 2011). Em particular, Grabner e Speckbacher (2016) indicam que uma percepção de falta de conhecimento sobre a relação de causa-e-efeito em uma dada tarefa faz com que gestores de organizações dependentes de criatividade atribuam maior confiança a controles de input na forma de processos de seleção de colaboradores.

Em geral, essas evidências sugerem ser importante considerar que sistemas de controle gerencial existem não apenas para afetar motivação extrínseca, mas também para facilitar o processo decisório ao capacitar colaboradores a alcançarem metas organizacionais (Sprinkle, 2003). Em particular, controles gerenciais podem também afetar motivação intrínseca e, desse modo, estimular criatividade (Cools et al., 2017; Davila \& Ditillo, 2017). Nesse sentido, maior nível de controle e desempenho em tarefas criativas não seriam objetivos necessariamente conflitantes, podendo co-existirem em determinados contextos e contribuirem conjuntamente para o alcance de metas organizacionais (Speckbacher, 2017). Importante também mencionar que organizações não dependentes de criatividade podem estabelecer controles gerenciais com o explícito propósito de inibir criatividade de seus colaboradores e, mesmo para aquelas que são dependentes de criatividade, é importante a inclusão de controles gerenciais que restrinjam a criatividade a um nível produtivo, evitando-se o problema de 'art for art's sake' (Grabner, 2014; Grabner \& Speckbacher, 2016).

\section{Sistemas de Controle Gerencial e Criatividade}

A pesquisa sobre criatividade tem buscado identificar fatores organizacionais, incluindo mecanismos de controle gerencial, que podem estimular criatividade (Shalley \& Gilson, 2004). Dentre os mecanismos de controle gerencial, a pesquisa em contabilidade gerencial tem dedicado especial atenção ao efeito de esquemas de incentivo sobre criatividade. Conforme comentado anteriormente, a pesquisa sobre criatividade em contabilidade gerencial tem seguido até o momento duas principais abordagens para tratar o tema. A primeira envolve os estudos de Williamson e seus coautores, enquanto a segunda inclui os estudos de Grabner. 
Nos estudos de Williamson e seus coautores, procura-se analisar como contratos de incentivo afetam desempenho criativo a partir de abordagem de agência. Dentre os principais resultados dos estudos iniciais, observa-se que desempenho em tarefas criativas é superior na presença de contratos de incentivo relativamente ao pagamento de salário fixo; no entanto, quando esse contrato de incentivo é baseado em uma medida de criatividade, o desempenho em tarefas criativas é inferior do que quando o contrato de incentivo é baseado em produtividade (Kachelmeier et al., 2008; Kachelmeier \& Williamson, 2010). Estudos posteriores ampliam essa perspectiva inicial ao examinar o papel de contratos de incentivo sobre criatividade em contexto de grupo (Chen et al., 2012) ou o papel de mecanismos adicionais de controle, tal como nível de dificuldade das metas (Webb et al., 2013) e se a meta é de input ou de output (Brüggen et al., 2018). Em estudo mais recente, Kachelmeier et al. (2019) examinam efeitos de diferentes contratos de incentivo sobre criatividade considerando dois estágios no processo de criatividade, um primeiro de preparação criativa e um segundo de incubação em que os ganhos decorrentes da etapa inicial são realizados. Nesse estudo, reforça-se a evidência dos estudos iniciais de que, quando se considera o estágio em que os ganhos são realizados, o desempenho em tarefas criativas é superior na presença de contratos de incentivo baseados em produtividade do que de salário fixo. Em geral, os estudos de Williamson e seus coautores sugerem ser possível utilizar mecanismos de controle gerencial, em particular, esquemas de incentivo, para estimular criatividade, contrário à hipótese de motivação intrínseca da criatividade.

Nos estudos de Grabner (2014; Grabner \& Speckbacher, 2016), procura-se examinar quais controles gerenciais são adotados por organizações dependentes de criatividade. Em particular, Grabner (2014) indica que organizações dependentes de criatividade fazem maior uso de remuneração baseada em desempenho para incentivar criatividade quando avaliação subjetiva de desempenho é usada de forma complementar. Em seu outro estudo, Grabner e Speckbacher (2016) examinam se maior confiança em controles gerenciais por parte de organizações dependentes de criatividade é explicado por fatores tais como motivação intrínseca e percepção de falta de conhecimento sobre a relação de causa-e-efeito em uma dada tarefa. De modo geral, os resultados de Grabner evidenciam que organizações dependentes de criatividade fazem uso de controles gerenciais para estimular criatividade, uma vez mais oferecendo evidências contrárias à existência de conflitos entre controle e criatividade.

Considerando os estudos adicionais em contabilidade gerencial, Speklé et al. (2017) oferecem evidências adicionais para a ausência de conflito entre controle e criatividade. Em particular, Speklé et al. (2017) empregam o modelo de alavancas de controle de Simons (1995) para demonstrar que intensidade de uso de mecanismos de controle gerencial aumentam a percepção de empoderamento de gestores o que, subsequentemente, resulta em efeitos positivos sobre criatividade. Cools et al. (2017) também empregam o modelo de alavancas de controle para demonstrar que orçamento pode, de um lado, estimular criatividade esperada quando usado de modo interativo por firmas criativas e, de outro lado, estimular criatividade responsiva quando usado de modo diagóstico. Desse modo, os resultados de Cools et al. (2017) indicam que mesmo o uso diagnóstico de mecanismos de controle gerencial pode estimular criatividade.

O estudo de Davila e Ditillo (2017) é outro que suporta a perspectiva de que mecanismos de controle gerencial podem facilitar ao invés de inibir o desempenho em tarefas criativas. De modo específico, em contextos de trabalho em grupo, Davila e Ditillo (2017) evidenciam que um conjunto de mecanismos de controle é utilizado com o propósito de delimitar o escopo de criatividade dos grupos de trabalho (mecanismos direcionais), enquanto outro conjunto é usado com o propósito de comunicar uma visão comum para os esforços criativos dos grupos de trabalho (sistemas inspiracionais). Vale destacar que controles direcionais seriam aqueles para lidar com o problema já mencionado de 'art for art's sake', enquanto controles inspiracionais estariam relacionados a controles informais. Davila e Ditillo (2017) destacam ainda o papel da estratégia para informar quais sistemas de controle gerencial são mais importantes para estimular criatividade de modo aderente às demandas estratégicas. 
De modo geral, percebe-se que a pesquisa sobre criatividade em contabilidade gerencial tem ampliado o seu escopo em termos dos mecanismos de controle examinados, deixando de enfatizar apenas o papel de esquemas de incentivo, mas incluindo perspectivas mais variadas de controle gerencial, com destaque para o modelo de alavancas de controle. Também merece atenção a ampliação do escopo desses estudos em termos das abordagens metodológicas, confiando-se tanto em estudos experimentais que têm a seu favor maior validade interna, mas que possuem limitações quanto à validade externa (Aguiar, 2017), quanto em estudos de levantamento que oferecem um melhor balanceamento entre validade interna e externa, sem deixar de mencionar os estudos qualitativos que permitem análises que levem em conta os contextos específicos das organizações para análise da relação entre controle e criatividade.

\section{Conclusão}

Considerando a ausência de pesquisas sobre criatividade em contabilidade gerencial no contexto brasileiro, são diversas as oportunidades existentes. Primeiro, criatividade no ambiente organizacional é percebida como importante fonte de vantagem competitiva (Shalley \& Gilson, 2004; Webb et al., 2013). Em particular, em contextos organizacionais em que criatividade é fonte de vantagem competitiva, tornase necessário um ambiente de trabalho que estimule criatividade (Grabner \& Speckbacher, 2016). Assim, estudos futuros poderiam examinar se no contexto brasileiro há diferenças em controles gerenciais usados por organizações dependentes de criatividade relativamente às demais organizações. Adicionalmente, para organizações não dependentes de criatividade, pode-se examinar se existem controles gerenciais usados especificamente com o propósito de inibir criatividade. Estudos com esses propósitos poderiam permitir um primeiro entendimento relativo à existência de controles gerenciais comumente usados no contexto brasileiro com a finalidade específica de estimular, delimitar ou inibir criatividade no ambiente de trabalho. Esses estudos poderiam empregar uma abordagem de contingência similar aos estudos de Grabner (2014; Grabner \& Speckbacher, 2016).

Segundo, uma razão para explicar a existência de conflito entre controle e criatividade é que controles gerenciais estão tipicamente associados à motivação extrínseca (Speckbacher, 2017). Enquanto esse argumento possa fazer sentido para controles formais de resultado cujo propósito principal é tipicamente o de restringir comportamentos, o mesmo pode não ocorrer quando se trata de controles informais e/ou de inputs que afetam comportamentos via auto-regulação (Ouchi, 1979). Por exemplo, Brüggen et al. (2018) mostram que metas informais e outras formas de comunicação informal podem contribuir para promover criatividade. Portanto, estudos no contexto brasileiro podem considerar o papel de controles gerenciais no desempenho de tarefas criativas para além de uma visão tradicional de mecanismos formais, diagnósticos e coercitivos ao explorar perspectiva mais abrangente que inclui controles informais, interativos e/ou facilitadores (Ouchi, 1979; Simons, 1995; Adler \& Borys, 1996). Estudos como os de Speklé et al. (2017) e Cools et al. (2017) já seguem essa perspectiva ao empregarem o modelo de alavancas de controle. Estudos futuros poderiam, de um lado, examinar efeitos desses diferentes tipos de controle sobre criatividade e, de outro lado, evidenciar a extensão com que, no contexto brasileiro, existe complementariedade no uso desses diferentes tipos de controle em organizações dependentes de criatividade. Esses estudos podem ser de particular relevância se considerarem as definições específicas de controles gerenciais e criatividade adotadas em determinada organização (Speckbacher, 2017). 
Terceiro, uma parte das pesquisas sobre criatividade em contabilidade gerencial enfatiza o efeito de contratos de incentivo sobre criatividade, sem considerar o processo pelo qual esse efeito ocorre (p. ex., Kachelmeier et al., 2008; Kachelmeier \& Williamson, 2010; Webb et al., 2013). Entretanto, outra parte passa a considerar esse processo ao incluir variáveis mediadoras (p. ex., Kachelmeier et al., 2019). Por exemplo, Chen et al. (2012) examinam se coesão de grupo explica o efeito dos tipos de incentivo sobre criatividade em um contexto de grupo. Em outro exemplo, Speklé et al. (2017) verificam se empoderamento explica o efeito da intensidade de uso das alavancas de controle sobre criatividade. Levando em conta uma perspectiva mais abrangente de controle gerencial mencionada anteriormente, pesquisas no contexto brasileiro podem procurar identificar processos pelos quais controles gerenciais afetam criatividade. Para identificar potenciais variáveis mediadoras relevantes, essas pesquisas podem confiar em estudos em psicologia e administração sobre criatividade-que já exploram o processo pelo qual criatividade é afetada-ao incluir variáveis tais como: motivação intrínseca (Eisenberger \& Rhoades, 2001), interesse por uma tarefa além de aspectos operacionais imediatos (operational ownership) (Dorenbosch, Engen \& Verhagen, 2005) e pressão por desempenho (Eisenberger \& Aselage, 2009). Alternativamente, estudos no contexto brasileiro podem considerar se variáveis mediadoras examinadas em outros contextos da pesquisa em contabilidade gerencial podem ser igualmente relevantes para a pesquisa sobre criatividade. Por exemplo, a literatura em contabilidade gerencial tem cada vez mais procurado entender o papel mediador de normas sociais para explicar efeitos comportamentais dos mecanismos de controle gerencial (p. ex., Cardinaels \& Yin, 2015; Maas \& Van Rinsum, 2013).

Por fim, criatividade tem sido tipicamente considerada como variável dependente no contexto da pesquisa em contabilidade gerencial (p. ex., Kachelmeier et al., 2008; Kachelmeier \& Williamson, 2010; Chen, et al., 2012; Webb et al., 2013). Nesse sentido, Speckbacher (2017) chama a atenção para uma oportunidade promissora de pesquisa referente à investigação do efeito conjunto de criatividade e controle sobre diferentes variáveis de resultado, tais como eficiência de processo, qualidade do produto, além de resultado financeiro. Em particular, criatividade é considerada como antecedente de processos inovativos (Axtell et al., 2000; Shalley \& Gilson, 2004). Estudos futuros podem verificar quais controles gerenciais reduzem ou potencializam eventuais efeitos positivos da criatividade sobre processos de inovação.

\section{Referências}

Adler, P. S., \& Borys, B. (1996). Two types of bureaucracy: Enabling and coercive. Administrative Science Quarterly, 61-89.

Adler, P. S., \& Chen, C. X. (2011). Combining creativity and control: Understanding individual motivation in large-scale collaborative creativity. Accounting, Organizations and Society, 36(2), 63-85.

Aguiar, A. B. (2017). Pesquisa experimental em contabilidade: propósito, desenho e execução. Advances in Scientific and Applied Accounting, 10(2), 224-244.

Amabile, T. M. (1982). Social psychology of creativity: A consensual assessment technique. Journal of Personality and Social Psychology, 43(5), 997-1013.

Amabile, T. M. (1983). The social psychology of creativity: A componential conceptualization. Journal of Personality and Social Psychology, 45(2), 357-376.

Amabile, T. M. (1996). Creativity in context: Update to" the social psychology of creativity." Westview press.

Amabile, T. M., \& Pillemer, J. (2012). Perspectives on the social psychology of creativity. The Journal of Creative Behavior, 46(1), 3-15. 
Axtell, C. M., Holman, D. J., Unsworth, K. L., Wall, T. D., Waterson, P. E., \& Harrington, E. (2000). Shopfloor innovation: Facilitating the suggestion and implementation of ideas. Journal of Occupational and Organizational Psychology, 73(3), 265-285.

Basadur, M., \& Finkbeiner, C. T. (1985). Measuring preference for ideation in creative problem-solving training. The Journal of Applied Behavioral Science, 21(1), 37-49.

Brüggen, A., Feichter, C., \& Williamson, M. G. (2018). The effect of input and output targets for routine tasks on creative task performance. The Accounting Review, 93(1), 29-43.

Cardinaels, E., \& Yin, H. (2015). Think twice before going for incentives: Social norms and the principal's decision on compensation contracts. Journal of Accounting Research, 53(5), 985-1015.

Chen, C. X., Williamson, M. G., \& Zhou, F. H. (2012). Reward system design and group creativity: An experimental investigation. The Accounting Review, 87(6), 1885-1911.

Cools, M., Stouthuysen, K., \& Van den Abbeele, A. (2017). Management control for stimulating different types of creativity: The role of budgets. Journal of Management Accounting Research, 29(3), 1-21.

Davila, A., \& Ditillo, A. (2017). Management control systems for creative teams: Managing stylistic creativity in fashion companies. Journal of Management Accounting Research, 29(3), 27-47.

Dorenbosch, L., Engen, M. L. V., \& Verhagen, M. (2005). On-the-job innovation: The impact of job design and human resource management through production ownership. Creativity and Innovation Management, 14(2), 129-141.

Eisenberger, R., \& Aselage, J. (2009). Incremental effects of reward on experienced performance pressure: Positive outcomes for intrinsic interest and creativity. Journal of Organizational Behavior: The International Journal of Industrial, Occupational and Organizational Psychology and Behavior, 30(1), 95-117.

Eisenberger, R., \& Cameron, J. (1996). Detrimental effects of reward: Reality or myth? American Psychologist, 51(11), 1153-1166.

Eisenberger, R., \& Rhoades, L. (2001). Incremental effects of reward on creativity. Journal of Personality and Social Psychology, 81(4), 728-741.

Eisenberger, R., \& Shanock, L. (2003). Rewards, intrinsic motivation, and creativity: A case study of conceptual and methodological isolation. Creativity Research Journal, 15(2-3), 121-130.

Grabner, I. (2014). Incentive system design in creativity-dependent firms. The Accounting Review, 89(5), 1729-1750.

Grabner, I., \& Speckbacher, G. (2016). The cost of creativity: A control perspective. Accounting, Organizations and Society, 48, 31-42.

Kachelmeier, S. J., Wang, L. W., \& Williamson, M. G. (2019). Incentivizing the creative process: From initial quantity to eventual creativity. The Accounting Review, 94(2), 249-266.

Kachelmeier, S. J., \& Williamson, M. G. (2010). Attracting creativity: The initial and aggregate effects of contract selection on creativity-weighted productivity. The Accounting Review, 85(5), 1669-1691.

Kachelmeier, S. J., Reichert, B. E., \& Williamson, M. G. (2008). Measuring and motivating quantity, creativity, or both. Journal of Accounting Research, 46(2), 341-373.

Maas, V. S., \& Van Rinsum, M. (2013). How control system design influences performance misreporting. Journal of Accounting Research, 51(5), 1159-1186.

Ouchi, W. G. (1979). A conceptual framework for the design of organizational control mechanisms. Management Science, 25(9), 833-848. 
Shalley, C. E., \& Gilson, L. L. (2004). What leaders need to know: A review of social and contextual factors that can foster or hinder creativity. The Leadership Quarterly, 15(1), 33-53.

Shields, M. D. (2015). Established management accounting knowledge. Journal of Management Accounting Research, 27(1), 123-132.

Simons, R. (1995). Levers of Control. Boston, MA, US: Harvard Business School Press.

Speckbacher, G. (2017). Creativity research in management accounting: A commentary. Journal of Management Accounting Research, 29(3), 49-54.

Speklé, R. F., van Elten, H. J., \& Widener, S. K. (2017). Creativity and control: A paradox-Evidence from the levers of control framework. Behavioral Research in Accounting, 29(2), 73-96.

Sprinkle, G. B. (2003). Perspectives on experimental research in managerial accounting. Accounting, Organizations and Society, 28(2-3), 287-318.

Webb, R. A., Williamson, M. G., \& Zhang, Y. M. (2013). Productivity-target difficulty, target-based pay, and outside-the-box thinking. The Accounting Review, 88(4), 1433-1457. 